\title{
THE FABER TRANSFORM AND ANALYTIC CONTINUATION ELGIN JOHNSTON
}

(Communicated by Irwin Kra)

Abstract. Let $\Omega \subseteq C$ be a bounded, simply connected domain, and let $\left\{\Phi_{n}(w)\right\}_{n=0}^{\infty}$ be the Faber polynomials associated with $\Omega$. Given $f(z)=$ $\sum_{k=0}^{\infty} c_{k} z^{k}$ analytic in $\Delta(0,1)$ we consider the function

$$
F(w)=\sum_{k=0}^{\infty} c_{k} \Phi_{k}(w) .
$$

We show that with proper restrictions on $\partial \Omega$, the existence of an analytic continuation of $f$ across a subarc of $C(0,1)$ implies the existence of an analytic continuation of $F$ across a subarc of $\partial \Omega$. Some converse results are also established.

1. Introduction. Let $\Omega \subseteq \mathbf{C}$ be a bounded, simply connected domain for which $\mathbf{C} \backslash \bar{\Omega}$ is connected. We use $g(\xi)$ to denote the unique function that is analytic and univalent on $\{|\xi|>1\}$, maps $\{|\xi|>1\}$ onto the exterior of $\Omega$, and has expansion

$$
g(\xi)=b_{-1} \xi+b_{0}+b_{1} / \xi+b_{2} / \xi^{2}+\cdots \quad\left(b_{-1}>0\right)
$$

in a neighborhood of $\infty$. The Faber polynomials associated with $\Omega$ (or $g$ ) are the polynomials $\left\{\Phi_{n}(w)\right\}_{n=0}^{\infty}$ determined by the following generating function relationship $[P]$ :

$$
\frac{g^{\prime}(\xi)}{g(\xi)-w}=\sum_{k=0}^{\infty} \Phi_{k}(w) \xi^{-k-1}
$$

It can be shown that with proper restrictions on $\partial D$ (see for example [S]) any function $F(w)$ analytic in $\Omega$ has a unique "Faber expansion"

$$
\sum_{k=0}^{\infty} c_{k} \Phi_{k}(w)
$$

that converges to $F(w)$ uniformly on compact subsets of $\Omega$. Conversely (again with appropriate restriction on $\partial \Omega$ ) any expression of the form (2) converges uniformly on compact subsets of $\Omega$ provided $\lim \sup _{k \rightarrow \infty} \sqrt[k]{\left|c_{k}\right|} \leq 1$.

In view of the last statement, we see that if

$$
f(z)=\sum_{k=0}^{\infty} c_{k} z^{k}
$$

is analytic on the unit disk $\Delta(0,1)$, then the function $F(w)=\sum_{k=0}^{\infty} c_{k} \Phi_{k}(w)$ might be analytic on $\Omega$. The operator $\mathscr{F}$ that takes a function (3) analytic on $\Delta(0,1)$ and maps it to the (formal) Faber series $\sum_{k=0}^{\infty} c_{k} \Phi_{k}(w)$ is called the Faber transform.

Received by the editors September 30, 1986 and, in revised form, March 18, 1987.

1980 Mathematics Subject Classification (1985 Revision). Primary 30B40; Secondary 30C99. 
In this paper we address some aspects of the following question: if $f(z)=$ $\sum_{k=0}^{\infty} c_{k} z^{k}$ is analytic on $\Delta(0,1)$ and has a certain property $P$, then to what extent is this property "inherited" by $F(w)=\mathscr{F}(f(z))(w)=\sum_{k=0}^{\infty} c_{k} \Phi_{k}(w)$ ? For example, it is clear that if $f(z)$ is a polynomial, then so is $\mathscr{F}(f(z))(w)$. Ellacott (and Gaier) [E] have shown that if $f(z)$ is a rational function then the same is true of $\mathscr{F}(f(z))(w) \ldots$ no restrictions on $\partial \Omega$ are required. In this paper we consider the question of analytic continuation. We show that with proper restriction on $\partial \Omega$ and/or on $f(z), \mathscr{F}(f(z))(w)$ has analytic continuation properties similar to those of $f(z)$.

In the remainder of the paper, $g(\xi)$ will be as defined in (1). We will also assume $b_{-1}=1 \ldots$ this assumption simply results in a change of scale and has no affect on the results.

2. $\partial \Omega$ analytic. As one would expect, the easiest case to consider is that in which $\partial \Omega$ is analytic.

THEOREM 1. Let $\partial \Omega$ be analytic and let $J \subseteq \partial \Omega$ be a subarc of $\partial \Omega$. For a given $f(z)$ analytic on $\Delta(0,1)$, the function $F(w)=\overline{\mathscr{F}}(f(z))(w)$ is analytic on $\Omega$ and has an analytic continuation across $J$ if and only if $f(z)$ has an analytic continuation across $g^{-1}(J)$.

Proof. The fact that $F(w)$ is analytic on $\Omega$ is well known (see $[\mathbf{S}]$ ). Since $\partial \Omega$ is analytic, $g(\xi)$ can be analytically and univalently continued to some domain $\left\{|\xi|>r_{0}\right\}$ for some $r_{0} \in(0,1)$. Hence if $f(z)=\sum_{k=0}^{\infty} c_{k} z^{k}$ on $\Delta(0,1)$, then for $r_{0}<|\xi|<1$ we have

$$
\begin{aligned}
F(g(\xi)) & =\sum_{k=0}^{\infty} c_{k} \Phi_{k}(g(\xi)) \\
& =\sum_{k=0}^{\infty} c_{k}\left(\xi^{k}+k \sum_{l=1}^{\infty} b_{k l} \xi^{-l}\right) \\
& =f(\xi)+\sum_{k=1}^{\infty} \sum_{l=1}^{\infty} k c_{k} b_{k l} \xi^{-1}
\end{aligned}
$$

where the coefficients $\left\{b_{k l}\right\}_{k, l=1}^{\infty}$ are the Grunsky coefficients associated with $g$ (see $[\mathbf{P}])$. Since $g(\xi)$ is analytic and univalent in $\left\{|\xi|>r_{0}\right\}$, it follows that for any $r \in\left(r_{0}, 1\right)$, we have $\left|b_{k l}\right|=o\left(r^{k+l}\right)$ as $k+l \rightarrow \infty$. Thus the last sum in (4) defines a function analytic in $\left\{|\xi|>r_{0}\right\}$. Hence, if $f(\xi)$ can be continued analytically across $g^{-1}(J) \subseteq\{|\xi|=1\}$, then the same is true of $F(g(\xi))$. Let $h(\xi)$ denote such an analytic continuation. Then for some open neighborhood $N\left(g^{-1}(J)\right) \subseteq\left\{|\xi|>r_{0}\right\}$ of $g^{-1}(J)$ we have $h(\xi)$ analytic on $\left\{r_{0}<|\xi|<1\right\} \cup N\left(g^{-1}(J)\right)$ and $h(\xi) \equiv F(g(\xi))$ on $\left\{r_{0}<|\xi|<1\right\}$. Define

$$
\tilde{F}(w)= \begin{cases}F(w) & (w \in \Omega), \\ h\left(g^{-1}(w)\right) & \left(w \in g\left(\left\{r_{0}<|\xi|<1\right\} \cap N\left(g^{-1}(J)\right)\right)\right) .\end{cases}
$$

Then $\tilde{F}(w)$ is an analytic continuation of $F$ across $J$.

Conversely, if $F(w)$ has an analytic continuation across $J$, then $F(g(\xi))$, defined for $r_{0}<|\xi|<1$, has an analytic continuation across $g^{-1}(J)$. It then follows from (4) that $f(z)$ has an analytic continuation across $g^{-1}(J)$. 
In view of Theorem 1 , we see that results concerning analytic continuations of functions analytic on $\Delta(0,1)$ have counter parts for functions defined by Faber expansions. For example, we have a "Faber-Hadamard" gap theorem.

COROLlaRY. Let $\partial \Omega$ be analytic and let

$$
F(w)=\sum_{k=1}^{\infty} c_{n_{k}} \Phi_{n_{k}}(w)
$$

be analytic in $\Omega$, but not on any neighborhood of $\bar{\Omega}$. Suppose there is a $\lambda>1$ such that the integer sequence $\left\{n_{k}\right\}_{k=1}^{\infty}$ satisfies $n_{k+1} / n_{k} \geq \lambda\left(k \geq k_{0}\right)$. Then $\partial \Omega$ is the natural boundary of $F$.

3. Nonanalytic $\partial \Omega$. If $\partial \Omega$ is not analytic, then there is no guarantee that $F(w)=\mathscr{F}(f(z))(w)$ defines a function analytic on $\Omega$. Something can be said, however, in the case where $\partial \Omega$ is a curve of bounded rotation and $f(z) \in A(\overline{\Delta(0,1)})=$ $\{f(z): f$ analytic on $\Delta(0,1)$, continuous on $\overline{\Delta(0,1)}\}$. Even if $\partial D$ is of bounded rotation and $f \in A(\overline{\Delta(0,1)})$ with

$$
f(z)=\sum_{k=0}^{\infty} c_{k} z^{k}
$$

it is possible that $\sum_{k=0}^{\infty} c_{k} \Phi_{k}(w)$ does not define a function in $A(\bar{\Omega})$. However if, in this case, we define

$$
F(w)=\mathscr{F}(f(z))(w)=\frac{1}{2 \pi i} \int_{\partial \Omega} \frac{f\left(g^{-1}(\xi)\right)}{\xi-w} d \xi \quad(w \in \Omega),
$$

then $F \in A(\bar{\Omega})$ and has Faber coefficients $\left\{c_{k}\right\}_{k=0}^{\infty}$ agreeing with the McLauren coefficients of $f$ (see $[\mathbf{G}]$ ). In this case we use the integral expression

$$
c_{k}=\frac{1}{2 \pi i} \int_{|z|=1} \frac{F(g(z))}{z^{n+1}} d z
$$

for the coefficients. We remark that it is easily checked that (5) agrees with the "coefficient transplant" description of $\mathscr{F}$ in the case when $\partial \Omega$ is analytic.

Thus if $\partial \Omega$ is of bounded rotation, then (5) defines a linear mapping

$$
\mathscr{F}: A(\overline{\Delta(0,1)}) \rightarrow A(\bar{\Omega}) .
$$

In fact, $\mathscr{F}$ is a continuous operator with respect to the supremum norms on $A(\overline{\Delta(0,1)})$ and $A(\bar{\Omega})$, with $\|\mathscr{F}\| \leq(1+2 V / \pi)$, where $V$ is the total rotation of $\partial \Omega$. (See $[\mathbf{G}]$.)

THEOREM 2. Let $\partial \Omega$ be of bounded rotation and let $f \in A(\overline{\Delta(0,1)})$. If $f(z)$ has an analytic continuation across the arc $I \subseteq C(0,1)$, then $F(w)=\mathscr{F}(f(z))(w)$ has an analytic continuation across $g(I)$.

PROOF. We first assume $I$ is a closed subarc of the unit circle, i.e. $I=\left\{e^{i \theta}: \alpha \leq\right.$ $\theta \leq \beta\}$. Let $h(z)$ be an analytic continuation of $f$ to $\Delta(0,1) \cup N(I)$ where $N(I)$ is some open neighborhood of $I$. Since $I$ is closed, we may assume $h$ is defined and continuous on $\overline{\Delta(0,1) \cup N(I)}$ and that $\mathbf{C} \backslash \overline{\Delta(0,1) \cup N(I)}$ is connected. By Mergelyan's Theorem [R] there is a sequence $\left\{P_{n}(z)\right\}_{n=1}^{\infty}$ of polynomials such that 
$P_{n}(z) \rightarrow h(z)$ uniformly on $\overline{\Delta(0,1) \cup N(I)}$. Now consider the sequence of polynomials

$$
Q_{n}(w)=\mathscr{F}\left(P_{n}(z)\right)(w) \quad(n=1,2,3, \ldots) .
$$

For $w \in \bar{\Omega}$ we have

$$
\begin{aligned}
\left|Q_{n}(w)-F(w)\right| & =\left|\mathscr{F}\left(P_{n}(z)\right)(w)-\mathscr{F}(f(z))(w)\right| \\
& \leq\|\mathscr{F}\|\left(\sup _{|z| \leq 1}\left|P_{n}(z)-f(z)\right|\right) \\
& =\|\mathscr{F}\|\left(\sup _{|z| \leq 1}\left|P_{n}(z)-h(z)\right|\right) \\
& \rightarrow 0 \text { as } n \rightarrow \infty .
\end{aligned}
$$

As equation (6) shows, $\left\{Q_{n}(w)\right\}_{n=1}^{\infty}$ converges uniformly to $F$ on $\bar{\Omega}$. We claim that $\left\{Q_{n}(w)\right\}_{n=1}^{\infty}$ also converges uniformly on $g(N(I) \cap\{|\xi| \geq 1\})$.

Given $w \in g(\overline{N(I)} \cap\{|z|>1\})$, find $\xi \in \overline{N(I)} \cap\{|z|>1\}$ with $w=g(\xi)$. Writing

$$
P_{n}(\xi)=\sum_{k=0}^{m_{n}} c_{k}^{(n)} \xi^{k}
$$

we have

$$
\begin{aligned}
Q_{n}(w) & =\sum_{k=0}^{m_{n}} c_{k}^{(n)} \Phi_{k}(w)=\sum_{k=0}^{m_{n}} c_{k}^{(n)} \Phi_{k}(g(\xi)) \\
& =\sum_{k=0}^{m_{n}} c_{k}^{(n)} \xi^{n}+\sum_{k=0}^{m_{n}}\left(k c_{k}^{(n)} \sum_{l=1}^{\infty} b_{k l} \xi^{-l}\right) \\
& =P_{n}(\xi)+\sum_{l=1}^{\infty}\left(\sum_{k=0}^{m_{n}} k c_{k}^{(n)} b_{k l}\right) \xi^{-l}
\end{aligned}
$$

Now

$$
R_{n}(\xi)=Q_{n}(g(\xi))-P_{n}(\xi)=\sum_{l=1}^{\infty}\left(\sum_{k=0}^{m_{n}} k c_{k}^{(n)} b_{k l}\right) \xi^{-l}
$$

is analytic in $|\xi|>1$ and vanishes at $\infty$. Furthermore, for a given real $\theta$,

$$
\lim _{\substack{\xi \rightarrow e^{i \theta} \\|\xi|>1}} R_{n}(\xi)=Q_{n}\left(g\left(e^{i \theta}\right)\right)-P_{n}\left(e^{i \theta}\right)
$$

Since $\left\{P_{n}\right\}$ is uniformly Cauchy on $\overline{\Delta(0,1)}$ and $\left\{Q_{n}(w)\right\}$ is uniformly Cauchy on $\bar{\Omega}$, it follows from the Maximum Modulus Theorem that $\left\{R_{n}(\xi)\right\}$ is uniformly Cauchy on $\{|\xi| \geq 1\}$. Now for $w=g(\xi) \in g(\overline{N(I)} \cap\{|z| \geq 1\})$, we have

$$
\begin{aligned}
\left|Q_{n}(w)-Q_{m}(w)\right| & =\left|Q_{n}(g(\xi))-Q_{m}(g(\xi))\right| \\
& \leq\left|P_{n}(\xi)-P_{m}(\xi)\right|+\left|R_{n}(\xi)-R_{m}(\xi)\right| .
\end{aligned}
$$

Since $\left\{P_{n}(\xi)\right\}$ is uniformly Cauchy on $\overline{\Delta(0,1) \cup N(I)}$ and $\left\{R_{n}(\xi)\right\}$ is uniformly Cauchy on $\{|\xi| \geq 1\}$, it follows that $\left\{Q_{n}(w)\right\}$ is uniformly Cauchy in $g(\overline{N(I)} \cap\{|\xi| \geq 1\})$. Combining with (6) we see that $\left\{Q_{n}(w)\right\}$ is uniformly Cauchy on $\bar{\Omega} \cup g(\overline{N(I)} \cap\{|\xi| \geq 1\}) \ldots$ the continuity of $g$ on $\{|\xi| \geq 1\}$ and the analyticity 
of $g$ on $\{|\xi|>1\}$ imply this last set is a neighborhood of the (closed) arc $g(I)$. Letting

$$
H(w)=\lim _{n \rightarrow \infty} Q_{n}(w)
$$

we have a function continuous on $\bar{\Omega} \cup g(\overline{N(I)} \cap\{|\xi| \geq 1\})$ and analytic on the interior of this set. Since this interior contains $g(I)$ and since $\left.H\right|_{\bar{\Omega}}=F, H$ is the desired analytic continuation.

Suppose now the subarc $I$ of the unit circle is not closed. Again let $N(I)$ be an open set containing $I$ with $\mathbf{C} \backslash(\Delta(0,1) \cup N(I))$ connected and suppose $h(z)$ is an analytic continuation of $f$ to $\Delta(0,1) \cup N(I)$. We write $I=\bigcup_{n=1}^{\infty} I_{n}$ where $\left\{I_{n}\right\}_{n=1}^{\infty}$ is an increasing sequence of closed subarcs of $I$. We can also find an increasing sequence $\left\{N\left(I_{n}\right)\right\}_{n=1}^{\infty}$ of open sets satisfying $I_{n} \subseteq N\left(I_{n}\right) \subseteq \overline{N\left(I_{n}\right)} \subseteq N\left(I_{n+1}\right)$ $(n=1,2, \ldots)$, with each $\mathbf{C} \backslash(\overline{\Delta(0,1)} \cup \overline{N(I)})$ connected and $\bigcup_{n=1}^{\infty} N\left(I_{n}\right)=N(I)$. Then $h_{n}(z)=\left.h\right|_{\Delta(0,1) \cup N\left(I_{n}\right)}$ is an analytic continuation of $f$ across $I_{n}$, and has a continous extension to $\overline{\Delta(0,1) \cup N\left(I_{n}\right)}$. As shown in the first part of the proof, we can find, for each $n$, a function $H_{n}(w)$ continuous on $\bar{\Omega} \cup g\left(N\left(I_{n}\right) \cap\{|\xi| \geq 1\}\right)$, analytic on the interior of this set and with $\left.H_{n}\right|_{\bar{\Omega}}=F$. Since

$$
\Omega \cup g(N(I) \cap\{|\xi| \geq 1\})=\bigcup_{n=1}^{\infty}\left[\Omega \cup g\left(N\left(I_{n}\right) \cap\{|\xi| \geq 1\}\right)\right]
$$

as an increasing union, we may define $H$ on $\Omega \cup(g(N(I) \cap\{|\xi| \geq 1\}))$ by

$$
H(w)= \begin{cases}F(\omega) & (w \in \Omega), \\ H_{n}(w) & \left(w \in g\left(N\left(I_{n}\right) \cap\{|\xi| \geq 1\}\right)\right) .\end{cases}
$$

It is clear that $H$ is well defined and analytic on $\Omega \cup\{g(N(I) \cap\{|\xi| \geq 1\})\}$ and hence is the desired analytic continuation.

If $\mathscr{F}^{-1}: A(\bar{\Omega}) \rightarrow A(\overline{\Delta(0,1)})$ is defined and continuous, then the converse of Theorem 2 holds. Unfortunately, the existence of $\mathscr{F}^{-1}$ is not automatic, even if $\partial \Omega$ is of bounded rotation. If $\partial \Omega$ is of bounded rotation, then $\mathscr{F}: A(\overline{\Delta(0,1)}) \rightarrow A(\bar{\Omega})$ is a one-to-one mapping, but may not be onto. In fact, given $F \in A(\bar{\Omega})$ ( $\partial \Omega$ of bounded rotation), one can assert that $F=\mathscr{F}(f)$ for some $f \in A(\overline{\Delta(0,1)})$ if and only if $h=F \circ g$ and its conjugate, $\tilde{h}$, are both continuous on $C(0,1)$ (see $[\mathbf{G}, \mathrm{p}$. 53]). In this case $F=\mathscr{F}(f)$ where

$$
f(z)=\frac{1}{2 \pi i} \int_{|\xi|=1} \frac{F(g(\xi))}{\xi-z} d \xi
$$

However, if $\mathscr{F}^{-1}: A(\bar{\Omega}) \rightarrow A(\overline{\Delta(0,1)})$ is defined (i.e. $\mathscr{F}: A(\overline{\Delta(0,1)}) \rightarrow A(\bar{\Omega})$ is onto), then $\mathscr{F}^{-1}$ is continuous by the Open Mapping Theorem [R].

THEOREM 3. Let $\partial \Omega$ be of bounded rotation and let $\mathscr{F}: A(\overline{\Delta(0,1)}) \rightarrow A(\bar{\Omega})$ be onto. Let $F(w) \in A(\bar{\Omega})$ and $J$ be a subarc of $\partial \Omega$. Then $F(w)$ has an analytic continuation across $J$ if and only if $f(z)=\mathscr{F}^{-1}(F(w))(z)$ has an analytic continuation across $g^{-1}(J)$.

Proof. The sufficiency was established in Theorem 2. The proof of the necessity is very similar so we may be somewhat brief. We consider the case in which $J$ is a "closed subarc" of $\partial \Omega \ldots$ that is $J=g\left(\left\{e^{i \theta}: \alpha \leq \theta \leq \beta\right\}\right)$ for some real $\alpha$ and $\beta$. 
The cases of "open" or "half-open" $J$ are then taken care of as in the last part of the proof of Theorem 2 .

Let $N(J)$ be an open set containing $J$ such that $\mathrm{C} \backslash \overline{\Omega \cup N(J)}$ is connected, $F(w)$ has an analytic continuation, $H(w)$, to $\Omega \cup N(J)$ and $H$ has a continuous extension to $\overline{\Omega \cup N(J)}$.

By Mergelyan's Theorem, there is a sequence $\left\{Q_{n}(w)\right\}$ of polynomials with $Q_{n}(w) \rightarrow H(w)$ uniformly on $\overline{\Omega \cup N(J)}$. Now each $P_{n}(z)=\mathscr{F}^{-1}\left(Q_{n}(w)\right)(z)$ is also a polynomial, and the continuity of $\mathscr{F}^{-1}$ implies that $P_{n}(z) \rightarrow f(z)=$ $\mathscr{F}^{-1}(F(w))(z)$ uniformly on $\overline{\Delta(0,1)}$. We claim that the sequence $\left\{P_{n}(z)\right\}$ is also uniformly convergent on $g^{-1}(\overline{N(J)} \backslash(\mathbf{C} \backslash \Omega))$. To see this write

$$
Q_{n}(w)=\sum_{k=0}^{m_{n}} c_{k}^{(n)} \Phi_{k}(w)
$$

as a Faber expansion. Then

$$
P_{n}(z)=\sum_{k=0}^{m_{n}} c_{k}^{(n)} z^{k}
$$

Let $z \in\{|\xi|>1\},|z|$ large. We can find $w \in \mathbf{C} \backslash \Omega\left(|w|\right.$ large) with $g^{-1}(z)=w$. Then

$$
\begin{aligned}
P_{n}(z) & =P_{n}\left(g^{-1}(w)\right)=\sum_{k=0}^{m_{n}} c_{k}^{(n)}\left(g^{-1}(w)\right)^{k} \\
& =\sum_{k=0}^{m_{n}} c_{k}^{(n)}\left[\Phi_{k}(w)+\sum_{l=1}^{\infty} d_{k l} w^{-l}\right] \\
& =Q_{n}(w)+\sum_{l=1}^{\infty}\left(\sum_{k=0}^{m_{n}} c_{k}^{(n)} d_{k l}\right) w^{-l}
\end{aligned}
$$

for some choice of coefficients $d_{k l}$ (see [G]). We note that

$$
R_{n}(z)=P_{n}(z)-Q_{n}(g(z))=\sum_{l=1}^{\infty}\left(\sum_{k=0}^{m_{n}} c_{k}^{(n)} d_{k l}\right)(g(z))^{-l}
$$

(as above, defined for $|z|$ large) can be continued analytically to $\{|z|>1\}$, and continuously to $\{|z| \geq 1\}$. Furthermore, $R_{n}(z)$ vanishes at $\infty$. Since $P_{n}(z)$ and $Q_{n}(g(z))$ are both uniformly Cauchy on $C(0,1)$ it follows from the Maximum Modulus Theorem that $\left\{R_{n}(z)\right\}$ is uniformly Cauchy on $\{|z| \geq 1\}$. From this point we may proceed as in the proof of Theorem 2 and assert that

$$
h(z)=\lim _{n \rightarrow \infty} P_{n}(z)=\left\{\begin{array}{l}
f(z) \quad(z \in \Delta(0,1)), \\
\lim _{n \rightarrow \infty}\left(R_{n}(z)+Q_{n}(g(z))\right) \quad\left(z \in g^{-1}(N(J) \backslash(\mathbf{C} \backslash \Omega))\right)
\end{array}\right.
$$

gives an analytic continuation of $f$ across $g^{-1}(J)$.

We conclude by noting that Theorem 3 allows us to state a "Faber-Hadamard" gap theorem for $A(\bar{\Omega})$.

COROLlARY. Suppose $\partial \Omega$ is of bounded rotation and $\mathscr{F}: A(\overline{\Delta(0,1)}) \rightarrow A(\bar{\Omega})$ is onto. Let $F(w) \in A(\bar{\Omega})$ have Faber series

$$
F(w) \sim \sum_{k=0}^{\infty} c_{n_{k}} \Phi_{n_{k}}(w)
$$


If $\lim \sup _{k \rightarrow \infty}\left|c_{n_{k}}\right|^{1 / n_{k}}=1$ and there is a number $\lambda>1$ with $n_{k+1} / n_{k}>\lambda$ $\left(k>k_{0}\right)$, then $\partial \Omega$ is the natural boundary for $F$.

\section{REFERENCES}

[E] S. W. Ellacott, On the Faber transform and efficient numerical rational approximation, SIAM J. Numer. Anal. 20 (1983), 989-1000.

[G] D. Gaier, Vorlesungen über Approximation im Komplexen, Birkhäuser, Basel, 1980.

[P] C. Pommerenke, Univalent functions, Vandenhoeck and Ruprecht, Göttingen, 1975.

[R] W. Rudin, Real and complex analysis, McGraw-Hill, New York, 1966.

[S] G. Schoeber, Univalent functions, Lecture Notes in Math., vol. 478, Springer-Verlag, Berlin and New York, 1975.

Department of Mathematics, Iowa State University, Ames, IOWA 50011 